\title{
Observation-Based Data Driven Adaptive Control of an Electromechanical Device
}

\author{
József K. Tar, Imre J. Rudas, Levente Kovács, Balázs Kurtán, Tamás Haidegger
}

\begin{abstract}
The model-based approach in control engineering works well when a reliable plant model is available. However, in practice, reliable models seldom exist: instead, typical "levels" of limited reliability occur. For instance, Computed Torque Control $(C T C)$ in robotics assumes almost perfect models. The Adaptive Inverse Dynamics Controller (AIDC) and the Slotine Li Adaptive Robot Controller (SLARC) assume absolutely correct analytical model form, and only allows imprecise knowledge regarding the actual values of the model parameters. Neglecting the effects of dynamically coupled subsystems, and allowing the action of unknown external disturbances means a higher level of corrupted model reliability. Friction-related problems are typical examples of this case. In the traditional control literature, such problems are tackled by either drastic "robust" or rather intricate "adaptive" solutions, both designed by the use of Lyapunov's $2^{\text {nd }}$ method that is a complicated technique requiring advanced mathematical skills from the designer. As an alternative design methodology, the use of Robust Fixed Point Transformations (RFPT) was suggested, which concentrates on guaranteeing the prescribed details of tracking error relaxation via generation of iterative control signal sequences that converge on the basis of Banach's Fixed Point Theorem. This approach is essentially based on the fresh data collected by observing the behavior of the controlled systems, rather than in the case of the traditional ones. For the first time, this technique is applied for order reduction in the adaptive control of a strongly nonlinear plant with significant model imprecisions: the control of a DC motor driven arm in dynamic interaction with a nonlinear environment is demonstrated via numerical simulations.
\end{abstract}

\section{INTRODUCTION}

A typical feature of the motion equations of strongly coupled nonlinear systems is that normally they do not have solutions in closed analytical form. The same holds for that of their controlled versions in which the controllers' feedback signals mean further couplings. "If the nominal trajectory that has to be tracked covers high dynamic range modellinearization around some working points, and subsequent application of the techniques elaborated for the control of Linear Time-Invariant (LTI) systems cannot provide satisfactory results, the application of truly nonlinear techniques becomes necessary. These techniques in general are based on Lyapunov's ingenious $2^{\text {nd }}$ method [1], [2] that (if applied in control technology) can guarantee the stability (sometimes global and asymptotic stability) of the motion of the controlled system without revealing any details of this motion. Such details as e.g., that of the trajectory tracking error relaxation, the appropriate (not necessarily "optimal") setting of the normally great number of arbitrary control parameters

\footnotetext{
${ }^{1}$ Antal Bejczy Center for Intelligent Robotics, Óbuda University, Budapest, Hungary \{tar.jozsef@nik., rudase, kovacs.levente@nik., haidegger@\}uni-obuda.hu, balazs.kurtan@gmail.com
}

are not directly addressed by this approach. It is definitely concentrating only on stability issues though it is extensively used for designing various adaptive controllers (e.g., [3], [4], [5], [6], [7], [8]).

In order to directly address the realization of a prescribed trajectory tracking error relaxation as primary design intent, as an alternative of Lyapunov's direct method, the RFPTbased adaptive control was suggested [9]. In this approach, this relaxation policy is prescribed via purely kinematic considerations. Without the application of adaptive techniques this policy could be exactly realized only in the possession of an exact system model and guaranteed lack of unknown external disturbances. Since normally we have only imprecise and incomplete system models and external disturbances are also present this policy can only be approximated. The concept of the "Response Function" was introduced in the control of these approximately modeled systems as follows: for the kinematically prescribed desired response (it is the "input argument" of this function) the controller calculates a control action in the possession of the approximate/incomplete model and exerts this action on the controlled system. Due to the modeling errors and the presence of unknown external disturbances the observed system response obtained for this control action (i.e., the "output" of this function) normally is different to the input. Instead tuning any model parameter as it happens in the case of the traditional adaptive controller or directly adjusting the control signals as e.g., in the Model Reference Adaptive Controllers (MRAC) the RFPT-based approach iteratively deforms the input of the response function until the output well approaches the desired response. The iterative sequence is generated by a contractive map and converges to the solution of the control task according to Banach's Fixed Point Theorem [10]. In comparison with the Lyapunov functionbased techniques, this new approach has the weak point that the iterative sequence can only provide a local basin of attraction around the appropriate solution. Leaving this basin may result in convergence to a useless fixed point, divergence to infinity, or some bounded chaotic "chattering".

In order to eliminate this weak point, the method was further investigated and refined. At the beginning, only the local properties of this transformation were investigated in the vicinity of the fixed point, and various tuning methods were suggested for keeping the control signal in the basin of attraction of the useful fixed point [11], [12]. Later its global properties were investigated in [13], [14], [15] and [16]. The conclusion of these investigations was that for a wide class of physical systems it was possible to so tune 
only one of the altogether three adaptive control parameters that monotone convergence to the useful fixed point could be maintained. If the tuned parameter exceeded a limit, the sequence still converged to the useful fixed point but it took oscillatory nature. These "precursor oscillations" generally can be observed by a simple, model-independent observer, therefore the tuning process can be kept at bay. As a consequence, the lack of guaranteed global stability was efficiently compensated from the point of view of practical applications. However, for the great majority of systems to be controlled, no complementary parameter tuning is required.

Another problem related to the use of the concept of response function may be related to the systems of higher order response. High order response typically occurs whenever the actuator drives a Classical Mechanical System through some deformable components. Normally, it is difficult to reliably observe higher order responses. However, by the development of a polynomial $4^{\text {th }}$ order differentiator the RFPT-based technique was successfully tested via simulation in the adaptive control of a $4^{\text {th }}$ order system [17]. In the control of LTI, or satisfactorily linearizable nonlinear systems, a practically useful technique is order reduction. This technique is based on the observation that the LTI systems can be tackled in the frequency picture where they can be described by complex fractional polynomials, called Transfer Functions. A fractional expression of two higher order polynomials can be approximated by that of two lower order ones. These well approximate the original ones in the frequency range of practical interest, and the controller can be designed by the use of this approximate model. More rigorously, this method is based on Padé's approximation theorem from 1892 [18] that is extensively used even in the control of non-integer order systems with long memory (e.g., [19]). However, this technique cannot be used for strongly nonlinear systems working in a wide dynamic range. The aim of the present paper is to show that the RFPT-based technique can be used for order reduction, even in the case of such systems. The paper is constructed as follows: in Section II the model of the system to be controlled is described. In Section III the application of the RFPT for order reduction in this case is described. Section IV contains the simulation results and their analysis. In Section V, the conclusions are drawn and the further planned work is outlined.

\section{The Model of The Plant to Be Controlled}

The plant is a DC-motor driven arm (via the application of gear reduction), and this arm is in dynamic interaction with a strongly nonlinear environment. The motor model and its appropriate parameters were taken from [20] as follows:

$$
\begin{gathered}
\ddot{q}=\frac{Q_{e}+Q_{e x t}-b \dot{q}-m g S \sin (\nu q)}{\Theta} \\
\dot{Q}_{e}=\frac{-R Q-K^{2} \dot{q}+K U}{L}
\end{gathered}
$$

where

- $q[\mathrm{rad}]$ denotes the rotational angle of the motor's axle,

- $Q_{e}[N \cdot m]$ is the torque of electromagnetic origin, exerted on the motor's axle,
- $Q_{\text {ext }}[N \cdot m]$ is the torque of external origin, acting on the motor's axle,

- $\nu=0.1$ is the gear reduction ratio,

- $R=1[\Omega]$ is the Ohmic resistance of the motor's coil system,

- $L=0.5[H]$ is its inductivity,

- $\Theta=0.01\left[\mathrm{~kg} \cdot \mathrm{m}^{2}\right]$ denotes the momentum of the rotary part of the motor,

- $b=0.1[N \cdot \mathrm{m} \cdot \mathrm{s} / \mathrm{rad}]$ describes the viscous friction of the motor's axle,

- $K=0 .[N m / A]$ is the motor's torque coefficient,

- $g=9.81\left[\mathrm{~m} / \mathrm{s}^{2}\right]$ denotes the gravitational acceleration,

- $S=0.05[\mathrm{~m}]$ is the length of the beam of the pendulum (with neglected momentum),

- $m=0.01[\mathrm{~kg}]$ is the mass of the point-like burden at the end of the pendulum,

- $U[V]$ denotes the control voltage.

Normally, $Q_{\text {ext }}$ depends on the properties of the environmental medium in dynamic interaction with the pendulum's axle. For modeling such interactions, various friction models were elaborated to describe e.g., the tire-road interaction ([21], [22], [23].) The "magic formula" suggested in [24], [25], [26] contains complicated trigonometrical functions, the Rill model [27] applies polynomials in connected bounded sections to describe the slipping-skidding phenomenon. (Its essence is that with the increase of the velocity in the beginning, the friction forces increase, but following a limit they begin to decrease.) Similar effects occur not only in the case of tire-road interaction: the stick-slip phenomenon means a challenge in control, since it frequently results in limit cycles in the motion of the controlled systems ([28], [29]). The identification of the friction models is also a very complicated task and normally it can be done only for a single degree of freedom system ([30], [31], [32]). Another practical problem is that the friction parameters depend on several factors (e.g., temperature), therefore real model-based control approach in this field does not seem to be practical. The idea of observed data based control seems to be more promising. Without the need of modeling any particular system in the simulations, the following model was used to describe the friction force acting on the moved work-piece:

$$
F_{w}=-\left(C_{w} \sigma_{2}\left(\nu \dot{q} / D_{w}\right)-A_{w} \frac{\left(\nu \dot{q} / D_{w}\right)^{3}}{\left(1+\left(\nu \dot{q} / B_{w}\right)^{4}\right)}\right.
$$

where $\sigma_{2}(x) \stackrel{\text { def }}{=} \frac{x}{1+|x|}, A_{w}=0.5[N], B_{w}=0.2[\mathrm{~s} / \mathrm{rad}]$, $C_{w}=0.5[N]$ and $D_{w}=0.2[\mathrm{~s} / \mathrm{rad}]$. This formula has the qualitative properties that are needed for the description of the stick-slip phenomenon. The torque of this force must be scaled by factor $\nu$ to describe its effect on the motor axle as $Q_{e x t}=\nu S F_{w}$. In he next section, the related control issues are discussed.

\section{Simultaneous AdAPtivity And ORder REDUCTION BY THE RFPT-BASED DESIGN}

Assume that $\left(\Lambda+\frac{d}{d t}\right) \int_{t_{0}}^{t}\left(q^{N}(\xi)-q(\xi)\right) d \xi \equiv 0$ is an "ideal formula" used to define the kinematically prescribed 
tracking error reduction $\left(\Lambda>0, q^{N}(t)\right.$ is the nominal, $q(t)$ is the actual trajectory). It leads to the desired $2^{\text {nd }}$ timederivative that corresponds to a PID-type control as:

$$
\begin{gathered}
\ddot{q}^{D e s} \stackrel{\text { def }}{=} \ddot{q}^{N}+\Lambda^{3} \int_{t_{0}}^{t}\left[q^{N}(\xi)-q(\xi)\right] d \xi \\
+3 \Lambda^{2}\left[q^{N}(t)-q(t)\right]+3 \Lambda\left[\dot{q}^{N}(t)-\dot{q}(t)\right]
\end{gathered},
$$

resulting fast exponential error-relaxation. In the given physical state of the system-determined by the actual $(q, \dot{q})$ at a given $Q_{e x t}$-the first equation of the group (1) could be used to determine the necessary electromagnetic torque $Q_{e}$ that would be necessary to guarantee the realization of $\ddot{q}^{D e s}$. Unfortunately, the $2^{\text {nd }}$ equation of the group tells us that $Q_{e}$ cannot be instantaneously set: by the application of an abrupt change in the control voltage $U$, only $\dot{Q}_{e}$ can be forced to vary abruptly. Consequently, in a precise control, (3) should be replaced by a policy that defines $\dddot{q}^{D e s}$, and the time-derivative of the first equation in the group should be calculated in order tho work with a $3^{\text {rd }}$ order controller.

To avoid this procedure, the RFPT-based order reduction can be formulated as follows: for fixed $\dot{q}$ the $2^{\text {nd }}$ equation of the group (1) describes a stable linear system that exponentially traces the abrupt jumps in $U$. If we assume that the electric components work far more quickly than the mechanical ones group (1) can be used for designing abrupt changes in $U$ to realize (3) in each control cycle. Of course, this is only an approximation that is not necessarily satisfactory. The role of the RFPT-based adaptive design consist in correcting this preliminary design, together with the effects of the modeling errors and unknown external disturbances. In this approach, $\ddot{q}^{D e s}$ is computed from (3), but instead of the "exact model" in (2) the following approximate environmental model is used: $\hat{F}_{w}=-C_{c} \nu \dot{q}$ with $C_{c}=300[\mathrm{Ns} / \mathrm{rad}]$. By the use of the first equation of the group (1), $Q_{e}^{D e s}$ is calculated for $\ddot{q}^{D e s}$. Assuming that $\dot{Q}_{e} \approx 0$ for a given constant $\dot{q}$ the stabilized value of the necessary $U^{D e s}$ is estimated from the $2^{\text {nd }}$ equation as:

$$
U^{D e s} \stackrel{\text { def }}{=} \frac{R}{K} Q_{e}^{\text {Des }}+K \dot{q} .
$$

The adaptivity is introduced in the above outlined argumentation when the $\ddot{q}^{D e s}$ value is replaced by its adaptively deformed counterpart for control cycle $n+1$ as

$$
\ddot{q}_{n+1}^{R e q} \stackrel{\text { def }}{=}\left(\ddot{q}_{n}^{R e q}+K_{c}\right)\left\{1+B_{c} \sigma\left(A_{c}\left[\ddot{q}_{n}-\ddot{q}_{n+1}^{D e s}\right]\right)\right\}-K_{c},
$$

in which $A_{c}, B_{c}$, and $K_{c}$ are adaptive control parameters, $\ddot{q}_{n}$ is the observed response at cycle $n$, and the function $\sigma(x)$ is defined as follows:

$$
\sigma(x) \stackrel{\text { def }}{=}\left\{\begin{array}{l}
-1 \text { if } x \leq-1, \\
x \text { if }-1<x<1, \\
1 \text { if } x \geq 1 .
\end{array}\right.
$$

Manifestly, if $\ddot{q}_{n}=\ddot{q}_{n+1}^{D e s}$, i.e., when we found the appropriate deformation, $\ddot{q}_{n+1}^{R e q}=\ddot{q}_{n}^{R e q}$, that is the solution of the control task is the fixed point of the mapping, defined in
(5). To achieve convergence, this mapping must be made contractive. For this purpose, normally, $B_{c}= \pm 1$, a very big $\left|K_{c}\right|$ and an appropriately small $A_{c}>0$ value have to be chosen. The useful and simple methodology for setting these parameters was published in several papers (e.g., [12], [16]). Therefore this issue will not be discussed in this paper. Briefly, for the idea of order reduction it can be stated that

- instead of using the frequency picture, it is directly formulated in the time-picture,

- instead of using any sophisticated tricks for function approximation, it applies a simple deformation principle,

- it simultaneously addresses modeling imprecisions, unknown external disturbances, and approximations reducing the complexity of the design process,

- it evades the need for the observation of higher order signals, therefore its operation is relatively very fast.

The latter property is very significant from the practical point of view: if a control strategy is based on the observation of higher order responses, its operation becomes very "clumsy": the adaptive correction manifests itself only after timeconsuming integration of time, during which the state of the controlled system can meander in dangerous ranges. In the next Section simulation results will be presented.

\section{Simulation Results}

The simulations were implemented by the use of SCILAB 5.4.1 for LINUX and its graphical tool called XCOS, that are freely down-loadable from the Web [33]. It is a software package developed for the needs of higher education in France [34] and it is widely used in France, India, China, and Japan [35]. It also is a useful tool for solving optimization problems [36].

The cycle time of the controller was set to be $\delta t=10^{-3} \mathrm{~s}$ that is satisfactory for Classical Mechanical Systems of relatively slow motion. For numerical integration Livermore Solver for Ordinary Differential Equations (LSodar) was chosen. It is a numerical solver providing an efficient and stable method to solve Ordinary Differential Equations (ODE) Initial Value Problems with automatic method switching for stiff and non-stiff problems and root-finding. It is a convenient solver that uses variable step size and combines the (Backward Differentiation Formula (BDF) and Adams integration methods. The stiffness detection is done by step size attempts with both methods. To achieve useful results, the allowable step-size was limited to $10^{-2}$ in setting the solver. The adaptive control parameters were set as $B_{c}=-1, K_{c}=$ $10^{4}$ and $A_{c}=10^{-5}$, and no tuning for $A_{c}$ was necessary. The RFPT-based approach has the convenient feature that for achieving good tracking no stiff trajectory tracking (i.e., large $\Lambda$ in (3) is needed). (The ordinary PID controllers need great value to achieve precise tracking therefore they may generate undesirable side-effects.) In our case, $\Lambda=2 s^{-1}$ was chosen for a nominal motion of comparable dynamic properties. The non-adaptive solution was absolutely useless. The trajectory tracking of the adaptive solution is shown in Fig. 1.

It can be observed that following a short transient partcaused by the inconsistency of the nominal and actual initial 

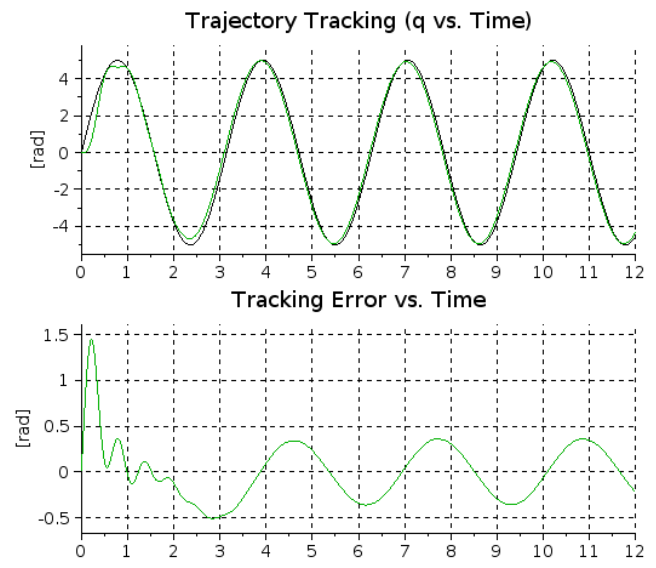

Fig. 1. The trajectory tracking of the adaptive controller: nominal (black line) and simulated (green line) trajectories for the motor axle vs. time $[s]$ (upper chart), and the trajectory tracking error (lower chart)

values - a smooth trajectory tracking was achieved. The chart of the phase trajectories in Fig. 2 reveals that the phase trajectory tracking is smooth and not any "chattering-type" anomaly occurred.

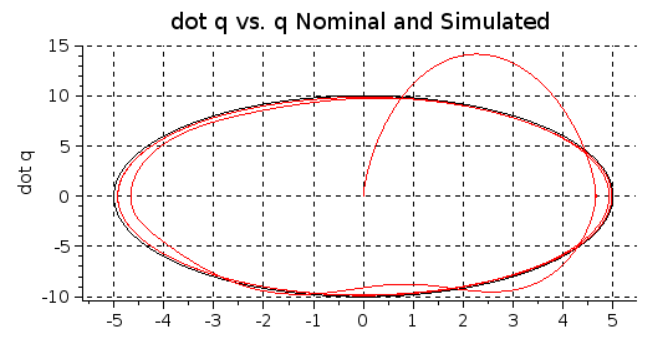

Fig. 2. Tracking of the phase trajectory (i.e. $\dot{q}$ vs. $q$ ) of adaptive controller: nominal (black line) and simulated (red line) phase trajectories for the motor axle vs. time $[s]$

Figure 3 reveals that the actual drag-force had increasing and decreasing segments with increasing velocity while the simple viscous model assumed only proportionality. Figure 4 exemplifies the the operation of the RFPT-based controller: the nominal, the desired (i.e. the nominal one corrected by the PID-type terms), and the realized/simulated $\ddot{q}$ values are in each other's close vicinity while the adaptively deformed "required" value significantly is different.

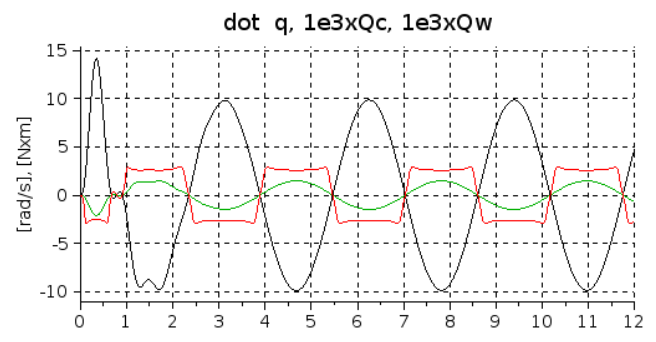

Fig. 3. The time-derivative of the motor axle $\dot{q}$ (black line), 1000 times the torque of the drag force by the environment, estimated by the use of the approximate model (green line) and the actual one (red line) vs. time $[s]$

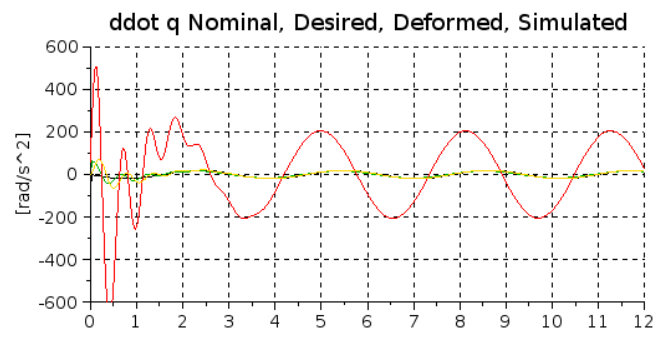

Fig. 4. The operation of the RFPT-based adaptivity: $\ddot{q}^{N}$ : black line, $\ddot{q}^{D e s}$ : green line, $\ddot{q}^{R e q}:$ red line and $\ddot{q}$ : ocher line vs. time $[s]$

The figures of the electromagnetic motor's torque (Fig. 5) and the control voltage (Fig. 6) reveal that in this casefollowing the relaxation of the initial transients- these control quantities remained almost of "sinusoidal nature", therefore in this case the environmental action was not too significant, and the adaptive controller mainly had to cope with the consequences of the order reduction.

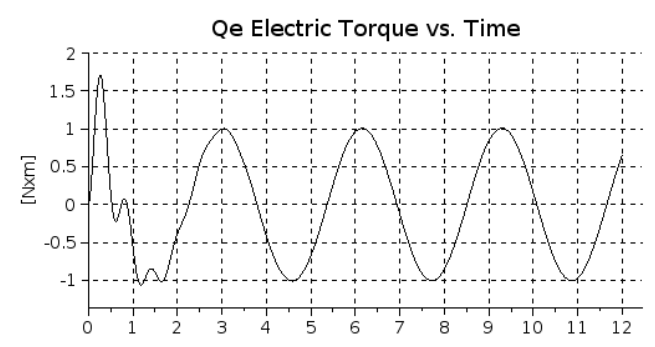

Fig. 5. The electric torque vs. time $[s]$

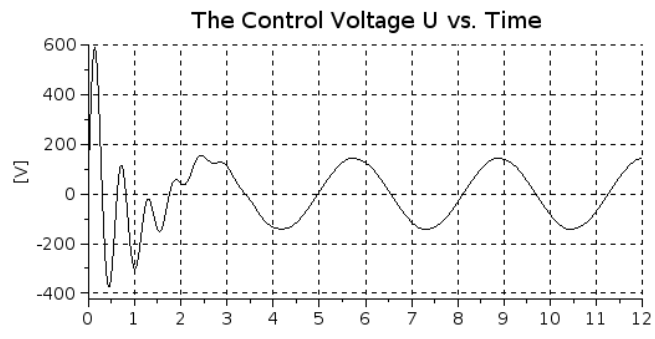

Fig. 6. The voltage $\mathrm{U}[\mathrm{V}]$ vs. time $[s]$

Further simulations were conducted to study what happens, if the weight of the environmental interactions increases. The following parameters were employed: $A_{w}=$ $30[N], B_{w}=0.2[\mathrm{~s} / \mathrm{rad}], C_{w}=30[\mathrm{~N}], D_{w}=0.2[\mathrm{~s} / \mathrm{rad}]$ and $C_{c}=600[\mathrm{Ns} / \mathrm{rad}]$. The nominal trajectory was also changed to avoid the huge initial transients related to the inappropriateness of the initial conditions. The control parameters were not modified. The appropriate trajectories reveal that the controller worked well even in this scenario (Figs. 7-12). 

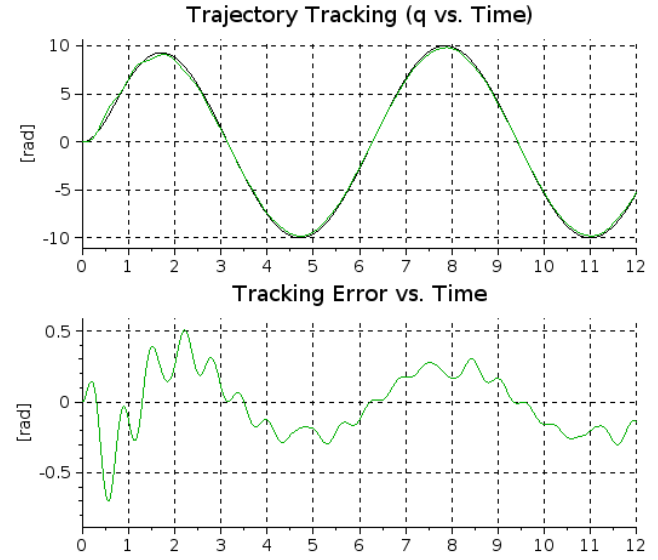

Fig. 7. The trajectory tracking of the adaptive controller for the modified task: nominal (black line) and simulated (green line) trajectories for the motor axle vs. time $[s]$ (upper chart) and the trajectory tracking error (lower chart)

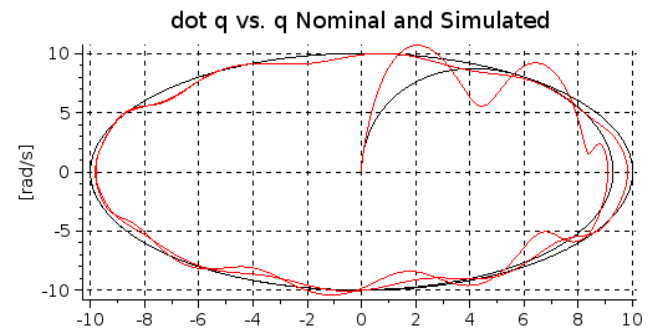

Fig. 8. Tracking of the phase trajectory (i.e. $\dot{q}$ vs. $q$ ) of adaptive controller for the modified task: nominal (black line) and simulated (red line) phase trajectories for the motor axle vs. time $[s]$

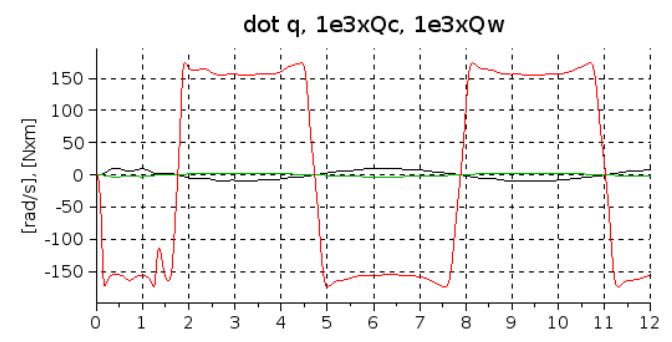

Fig. 9. The time-derivative the motor axle for the modified task $\dot{q}$ (black line), 1000 times the torque of the drag force by the environment estimated by the use of the approximate model (green line) and the actual one (red line) vs. time $[s]$

\section{CONCLUSIONS}

In this paper, a Robust Fixed Point Transformationsbased adaptive technique was applied to control an electromechanical device in strong dynamic interaction with an unreliably and roughly modeled environment. Though, from the mathematical point of view, this task would be a $3^{\text {rd }}$ order problem, in this approach it was reduced to a $2^{\text {nd }}$ order problem. All trajectories were successfully tackled by the adaptive technique suggested. In contrast to the traditional order reduction techniques that mainly are applicable for LTI system in the frequency domain, the present technique aims at the time domain. The adaptive capabilities of the
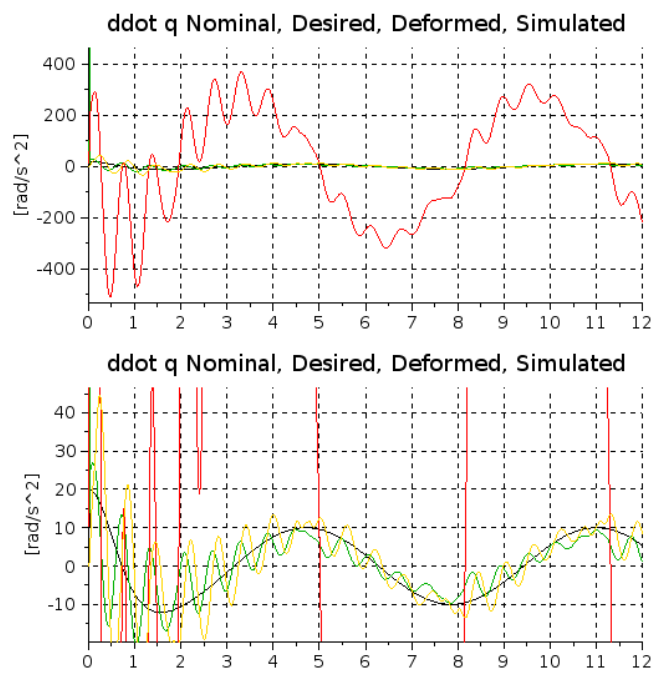

Fig. 10. The operation of the RFPT-based adaptivity for the modified task: $\ddot{q}^{N}$ : black line, $\ddot{q}^{D e s}$ : green line, $\ddot{q}^{R e q}$ : red line and $\ddot{q}$ : ocher line vs. time $[s]$

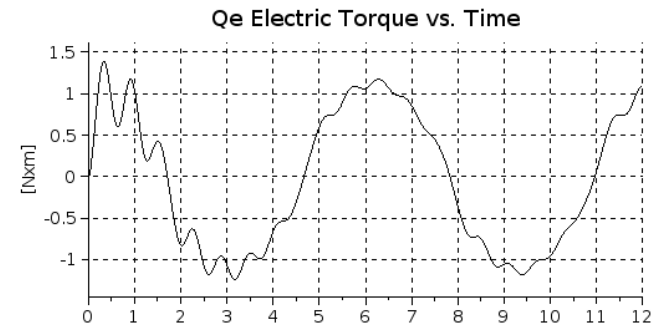

Fig. 11. The electric torque vs. time $[s]$ for the modified task

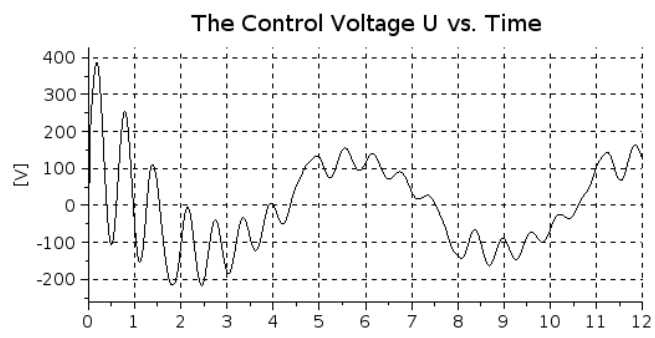

Fig. 12. The voltage $\mathrm{U}[\mathrm{V}]$ vs. time $[s]$ for the modified task

design simultaneously correct the consequences of the order reduction, and compensate for the unknown environmental interactions. This statement was substantiated by two sets of simulations. First, the effects of the order reduction dominated the problem, while in the second case, the significance of the external disturbances was increased.

The main feature of this technique is that in contrast to the model-based controllers it is rather based on approximate models that are neither identified nor amended. Instead of that the effects of their deficiencies are compensated by the use of fresh, observation-based data characteristic to the "response function". This means that the present technique 
needs more observation data than the feedback needs of the more traditional solutions.

In the next step, we plan to implement the possible application of our method is surgical operations, were normally individual tissue models should be applied for each patient to describe friction and sticking/slipping phenomena. For these cases, the existence of easily available, identifiable, and reliable models cannot be expected [37]. Our experiments will be focusing on the empirical validation of the control algorithms based on an electric motor-driven rod poking on artificial tissue phantoms.

\section{ACKNOWLEDGMENT}

The authors thankfully acknowledge the financial support provided by TÁMOP-4.2.2.A-11/1/KONV-2012-0012: Basic research for the development of hybrid and electric vehicles. The project is supported by the Hungarian Government, and co-financed by the European Social Fund. Our work has been partially supported by the Hungarian Scientific Research Fund OTKA K-106392. T. Haidegger is a Bolyai Fellow of the Hungarian Academy of Sciences.

\section{REFERENCES}

[1] A.M. Lyapunov. A general task about the stability of motion. (in Russian). Ph.D. Thesis, University of Kazan, 1892.

[2] A.M. Lyapunov. Stability of motion. Academic Press, New-York and London, 1966.

[3] R. Isermann, K.H. Lachmann, and D. Matko. Adaptive Control Systems. Prentice-Hall, New York DC, USA, 1992.

[4] Jean-Jacques E. Slotine and W. Li. Applied Nonlinear Control. Prentice Hall International, Inc., Englewood Cliffs, New Jersey, 1991.

[5] R.M. Murray, Z. Li, and S.S. Sastry. A mathematical introduction to robotic manipulation. CRC Press, New York, 1994.

[6] C.C. Nguyen, S.S. Antrazi, Zhen-Lei Zhou, and C.E. Campbell Jr. Adaptive control of a stewart platform-based manipulator. Journal of Robotic Systems, 10(5):657-687, 1993.

[7] J. Somló, B. Lantos, and P.T. Cát. Advanced Robot Control. Akadémiai Kiadó, Budapest, 2002.

[8] K. Hosseini-Suny, H. Momeni, and F. Janabi-Sharifi. Model reference adaptive control design for a teleoperation system with output prediction. J Intell Robot Syst, DOI 10.1007/s10846-010-9400-4:1-21, 2010.

[9] J.K. Tar, J.F. Bitó, L. Nádai, and J.A. Tenreiro Machado. Robust Fixed Point Transformations in adaptive control using local basin of attraction. Acta Polytechnica Hungarica, 6(1):21-37, 2009.

[10] S. Banach. Sur les opérations dans les ensembles abstraits et leur application aux équations intégrales (About the Operations in the Abstract Sets and Their Application to Integral Equations). Fund. Math., 3:133-181, 1922.

[11] J.K. Tar. Towards replacing Lyapunov's 'direct' method in adaptive control of nonlinear systems (invited plenary lecture). In Proc. of the Mathematical Methods in Engineering Intl. Symp. (MME), Coimbra, Portugal, 2010.

[12] J.K. Tar, L. Nádai, I.J. Rudas, and T.A. Várkonyi. RFPT-based adaptive control stabilized by fuzzy parameter tuning. In Proc. of the $9^{\text {th }}$ European Workshop on Advanced Control and Diagnosis (ACD 2011), Budapest, Hungary, pages 1-8, 2011.

[13] T.A. Várkonyi, J.K. Tar, I.J. Rudas, and I. Krómer. VS-type stabilization of MRAC controllers using robust fixed point transformations. In Proc. of the $7^{\text {th }}$ IEEE Intl. Symp. on Applied Computational Intelligence and Informatics (SACI), 2012, Timişoara, Romania, pages 389-394, 2012.

[14] K. Kósi, Sz. Hajdu, J.F. Bitó, and J.K. Tar. Chaos formation and reduction in Robust Fixed Point Transformations based adaptive control. In Proc. of the 4th IEEE Intl. Conf. on Nonlinear Science and Complexity (NSC 2012), Budapest, Hungary, pages 211-216, 2012.
[15] K. Kósi, Á. Breier, and J.K. Tar. Chaos patterns in a 3 degree of freedom control with robust fixed point transformation. In Proc. of the $13^{\text {th }}$ IEEE Intl. Symp. on Computational Intelligence and Informatics, Budapest, Hungary, pages 1-5, 2012.

[16] K. Kósi, J.K. Tar, and I.J. Rudas. Improvement of the stability of RFPT-based adaptive controllers by observing "precursor oscillations". In Proc. of the $9^{\text {th }}$ IEEE Intl. Conf. on Computational Cybernetics, Tihany, Hungary, pages 267-272, 2013.

[17] K. Kósi, T.A. Várkonyi, J.K. Tar, I.J. Rudas, and J.F. Bitó. On the simulation of RFPT-based adaptive control of systems of 4th order response. In Proc. of the IEEE $11^{\text {th }}$ Intl. Symp. on Intelligent Systems and Informatics (SISY 2013), September 26-28, 2013, Subotica, Serbia, pages 259-264, 2013.

[18] H. Padé. Sur la répresentation approchée d'une fonction par des fractions rationelles (Thesis). Ann. École Nor. (3), 9, 1892, pp. 1-93 supplement, 1892.

[19] R.S. Barbosa and J.A. Tenreiro Machado. Implementation of discretetime fractional-order controllers based on LS approximations. Acta Polytechnica Hungarica, 3(4):5-22, 2006.

[20] http://ctms.engin.umich.edu/CTMS/index.php?example= MotorSpeed\&section=SystemModeling. Last time chechked: Feb. 1 2014.

[21] T.D. Gillespie. Fundamentals of Vehicle Dynamics (1st edition). SAE International, 1992.

[22] E. Bakker, H.B. Pacejka, and L. Lidner. A new tyre model with an application in vehicle dynamics studies. SAE Paper 890087, pages 615-619, 1989.

[23] A. Zanten, R. Erhardt, and A. Lutz. Measurement and simulation of transients in longitudinal and lateral tire forces. SAE Paper 900210 , 99(6):300-318, 1990.

[24] H.B. Pacejka and E. Bakker. The magic formula tyre model. Vehicle System Dynamics, 21:1-18, 1993.

[25] J.J.M. van Oosten and E. Bakker. Determination of magic tyre model parameters. Vehicle System Dynamics, 21:19-29, 1993.

[26] L. Lidner. Experience with the magic formula tyre model. Vehicle System Dynamics, 21:30-46, 1993.

[27] G. Rill. Simulation von Kraftfahrzeugen. Vieweg+Teubner Verlag, 1994.

[28] B. Armstrong-Helouvry. Stick slip and control in low-speed motion. IEEE Trans. on Automatic Control, 38(10):1483-1496, 1990.

[29] Seung-Jean Kim and In-Joong Ha. On the existence of caratheodory solutions in mechanical systems with friction. IEEE Trans. on Automatic Control, 44(11):2086-2089, 1999.

[30] Seung-Jean Kim, Sung-Yeol Kim, and In-Joong Ha. An efficient identification method for friction in single-dof motion control systems. IEEE Trans. on Control Systems Technology, 12(4):555-563, 2004.

[31] L. Márton and B. Lantos. Identification and model-based compensation of striebeck friction. Acta Polytechnica Hungarica, 3(3):45-58, 2006.

[32] B. Takarics. TP Model Transformation Based Sliding Mode Control and Friction Compensation (Ph.D. dissertation). Budapest University of Technology and Economics, 2011.

[33] http://www.scilab.org. Last time chechked: Feb. 12014.

[34] http://www.scilab.org/education/higher_education.

[35] http://www.scilab.org/communities/international/.

[36] M. Baudin ${ }^{1}$, V. Couvert ${ }^{1}$, and S. Steer ${ }^{2}$. Optimization in SCILAB. ${ }^{1}$ Scilab Consortium, ${ }^{2}$ INRIA Paris - Rocquencourt, 2010.

[37] Á. Takács, S. Jordán, R.-E. Precup, L. Kovács, I. Rudas, and T. Haidegger. Review of tool-tissue interaction models for robotic surgery applications. In Proc. of the $12^{\text {th }}$ IEEE Intl. Symposium on Applied Machine Intelligence and Informatics (SAMI), Herlány, pages 339-344, 2014. 